\title{
Photobiological Basics of Photomedicine: A Work of Art Still in Progress
}

R Glen Calderhead

Clinique L Dermatology, Goyang, Korea

Received December 6, 2017

Accepted December 10, 2017

\section{Correspondence}

R Glen Calderhead

Clinique L Dermatology, 219 Sowon-ro, Haengsindong, Deogyang-gu, Goyang 10534, Korea

Tel.: +82-70-4714-6019

Fax: $+82-1600-3660$

E-mail: docrgc12132igmail.com

(C) Korean Society for Laser Medicine and Surgery

(c) This is an open access article distributed under the terms of the Creative Commons Attribution NonCommercial License (http://creativecommons.org/ licenses/by-nc/4.0) which permits unrestricted noncommercial use, distribution, and reproduction in any medium, provided the original work is properly cited.
The medical laser has been in use for only 57 years. In that comparatively short period of time, the field has expanded dramatically to embrace almost every medical speciality, harvesting the unique power of laser energy to achieve significant clinical effects through the science of lasertissue interactions. The vast range of reactions includes incision, excision, vaporization, and coagulation of tissue in the surgical field, which are all governed by the photobiological basics for laser-tissue interactions. On the other hand, in the late 1970s, initially defocused surgical lasers and then dedicated low output lasers were used in "laser biostimulation' to control pain and heal ulcers and other wounds in what became known as 'low level laser therapy' (LLLT). The advent of the NASA light-emitting diode (LED) in the late 1990s gave researchers and clinicians a new non-laser phototherapeutic light source that has led to 'low level laser therapy' to be renamed 'low level LIGHT therapy', even though the acronym, LLLT, remains the same. Another non-laser device, the intense pulsed light (IPL) system, was added to the armamentarium of light-based medicine in the late 1990s, delivering a milliseconddomain pulse of high-powered polychromatic light spanning from the near infrared region to the shorter visible wavelengths. IPL systems are equipped with cut-off filters to permit semiselectivity in pigmented targets, allowing the treatment of vascular lesions, pigmented lesions, and hair removal. Although they are all different technologies, lasers, LEDs, and IPL systems all contain the magic letter ' $L$ '- light. They are all governed by the laws that govern light and are dependent on the same photobiological basics. This article explores these basics, and reports that although systems have developed in sophistication and scope, the basic understanding of light-tissue interactions is still the greatest tool the practitioner in light-based medicine and surgery can possess.

\section{Key words}

Photosurgery; Photobiomodulation; Light-tissue interaction 


\section{THE HISTORY}

Almost every article, book and presentation on laser seems to start off by telling us all something we already know, namely that "LASER is the acronym for Light Amplification by Stimulated Emission of Radiation", closely followed by the already exhaustively reported successful oscillation of a laser by Theodore Maiman in April of 1960 based on a ruby crystal pumped by a flashlamp.' In fact, the background steps which led to this 1960 event began 60 years earlier.

Max Plank in 1990 held that photons had a duality of both particle and waveform, and light was thus part of the electromagnetic spectrum. This led to the theory from Albert Einstein in 1916 that an excited atom could return spontaneously to its ground state of energy thereby emitting a photon at a particular wavelength, and then in 1917 he argued that it should be possible to stimulate photon emission from already excited electrons in a medium by collision with these spontaneously-emitted photons, ${ }^{2}$ although he never discussed the potential for amplification. Stimulated emission was not practically demonstrated until 1928 by Landenberg, and the state of population inversion, by which a greater number of excited atoms could exist in a medium than unexcited ones, was suggested by Fabrikant in 1940 which pointed towards the concept of amplification. ${ }^{3}$ Charles $H$ Townes first showed amplification of microwave radiation by stimulated emis- sion, the MASER, in 1951, and produced a preliminary lab notebook sketch of an "optical MASER" in 1957. The first recorded written definition of a LASER derived from 'light amplification by stimulated emission' by Gordon Gould followed in the same year, 3 years before the operation of such a laser was successfully demonstrated. ${ }^{4}$ In 1958 , Townes together with Arthur Schawlow published the first detailed paper describing the components of the optical MASER': ${ }^{5}$ the race was then on in earnest across the scientific world to develop the first practical laser system and as we all know, the race was won by Maiman.

The steps towards the successful demonstration of laser energy may have taken over 60 years, but once it was shown to be practical, a very large number of lasers based on different media very quickly followed. In the first few years after Maiman's pulsed $694.3 \mathrm{~nm}$ ruby laser, many hundreds of substances were found to be capable of delivering laser energy but of them all a very few lasers first used in surgery and medicine emerged and stood the test of time, as listed in Table $1 .{ }^{6}$

By 1970, the laser had gone from the realms of the laboratory, and the more esoteric and Sci-Fi military potential applications, into medical indications with the first recorded use of the medical laser being by dermatologist, researcher and laser pioneer, Professor Leon Goldman (1906-1997), in the early 1960s with the establishment of his laser laboratory in the University of Cincinnati. Goldman saw the potential for the selective uptake of

Table 1. Timeline for medical laser development, and non-laser sources

\begin{tabular}{|c|c|c|c|c|}
\hline Year & Medium & Medium type & Wavelength (s) (main line [s]) & Beam mode \\
\hline \multirow[t]{2}{*}{1960} & Ruby (Ru) & Solid state crystal & $694.3 \mathrm{~nm}$ & Pulsed \\
\hline & Helium/neon (HeNe, type 1) & Gas & $14000 \mathrm{~nm}(1.4 \mu \mathrm{m})$ & CW \\
\hline 1961 & $\begin{array}{l}\text { Neodymium-doped yttrium aluminium garnet (Nd:YAG) } \\
\text { Er:YAG, Ho:YAG }\end{array}$ & Solid state crystal & $\begin{array}{l}1064 \text { nm (Er:YAG, } 2940 \text { nm; } \\
\text { Ho:YAG, } 2010 \mathrm{~nm})\end{array}$ & Pulsed \\
\hline \multirow[t]{2}{*}{1962} & Gallium arsenide (GaAs) & Solid state laser semiconductor (diode) & $670 \mathrm{~nm}, 709 \mathrm{~nm}, 904 \mathrm{~nm}$ & Pulsed \\
\hline & HeNe (type 2) & Gas & $632.8 \mathrm{~nm}$ & CW \\
\hline \multirow[t]{3}{*}{1964} & Carbon dioxide $\left(\mathrm{CO}_{2}\right)$ & Gas & $10600(1.06 \mu \mathrm{m})$ & \\
\hline & CW Nd:YAG & Solid state crystal & $1064 \mathrm{~nm}, 1320 \mathrm{~nm}$ & Quasi-CW \\
\hline & Argon ion $(\mathrm{Ar})$ & Gas & $488 \mathrm{~nm}, 514.5 \mathrm{~nm}$ & CW \\
\hline 1965 & Frequency-doubled Nd:YAG & Solid State Crystal & $532 \mathrm{~nm}$ & Quasi-CW \\
\hline \multirow[t]{2}{*}{1966} & Metal vapour (Cu, Au, etc.) & Vaporized metal & $\begin{array}{l}\text { Cu: } 510.6,578.2 \\
\text { Au: } 627.8\end{array}$ & Quasi-CW \\
\hline & Rhodamine & Liquid dye & Tunable (560-635 nm) & Pulsed \\
\hline \multirow[t]{2}{*}{1970} & Xenon dimer $\left(\mathrm{Xe}_{2}\right)^{\prime}$ 'Excimer laser' & Gas & $172 \mathrm{~nm}$ & Pulsed \\
\hline & Gallium aluminium arsenide (GaAlAs) & Solid state laser diode & $810 \mathrm{~nm}, 830 \mathrm{~nm}$ & $\mathrm{CW}$ \\
\hline \multicolumn{5}{|c|}{ Main non-laser sources } \\
\hline 1961 & Near infrared light-emitting diode (LED) & Solid state diode & $\approx 790 \mathrm{~nm}$ & CW \\
\hline 1962 & Visible LED & Solid state diode & $\approx 670 \mathrm{~nm}$ & CW \\
\hline 1991 & Intense pulsed light (IPL) & Polychromatic flashlamp & $\approx 400 \mathrm{~nm}-1400 \mathrm{~nm}$ & Pulsed \\
\hline
\end{tabular}

Pulsed, pulse width $\leq 1.0 \mathrm{~ms}$; Quasi-CW, pulse train with frequencies $\geq \mathrm{kHz}$ domain. 
blue-green continuous wave argon laser energy in both vascular and melanogenic pigmented lesions, ${ }^{6}$ whereas the carbon dioxide $\left(\mathrm{CO}_{2}\right)$ laser was seized upon for surgical indications owing to its ability to be used as a "laser scalpel', offering a dry field through its hemostatic attributes and mostly pioneered by Professor Isaac Kaplan in Israel (1919-2012). The ruby and argon lasers also found early indications in the mid 1960s in ophthalmological applications with pioneers such as Professor Francis A L'Esperance, Jr (1931 ) in transcorneal retinal surgery to weld detached retinas and coagulate proliferating blood vessels to prevent vison loss in patients with diabetic retinopathy. ${ }^{8}$ The $\mathrm{CO}_{2}$ laser, interestingly, found an application in ophthalmology as the first system used in 1968 for refractive radial keratotomy, reshaping the cornea with a series of radial mini-incisions in the cornea to correct vision, but it was superseded in 1973 by the excimer laser. L'Esperance perfected the excimer technique and in addition to being the first ophthalmologist to use the argon laser in a human eye, he was the first to develop the more effective refractive keratoplasty technique, literally sculpting the cornea with an excimer laser, and the predecessor of the Lasik procedure.

However in the first decade or so of the laser's incursion into the medical field, the majority of both manufacturers and clinicians looked on the laser more as a solution in search of a problem, rather than considering what it could be really used for based on photobiological science and use of parameters. Indeed many of the 'expert' clinicians who adopted the laser simply 'because it was there' condemned the laser as being 'unsafe' due to the sometimes disastrous results they obtained. Why then were the real pioneers so successful compared to the majority who were not? Simply because the pioneers empirically understood and applied the pure science of light-tissue interaction whereby different beam modes, pulsed or

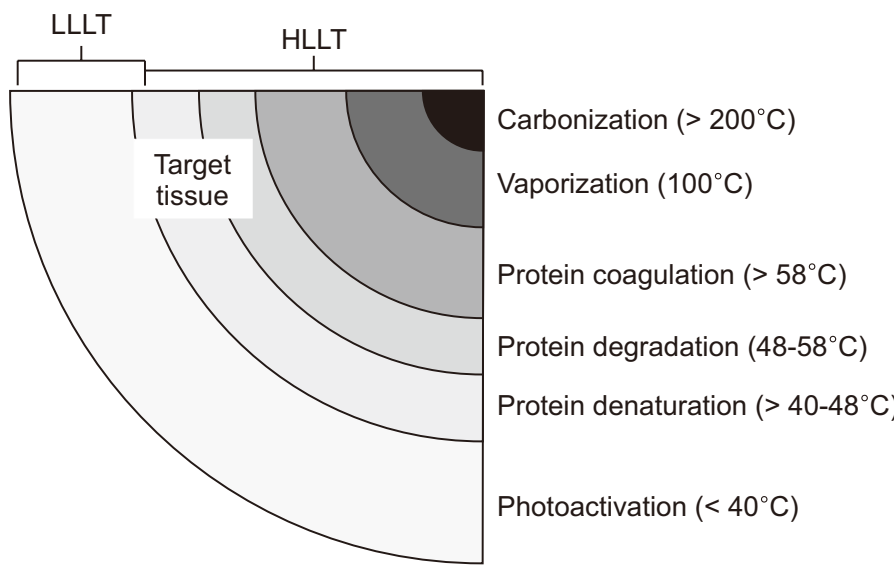

CW, and different wavelengths could do different things. To put this simply, their successful indication of the laser in their various fields was based on their knowledge and understanding of the photobiological basics which govern both photosurgery and phototherapy, and these comprise the next section.

\section{PHOTOBIOLOGY OF LIGHT-TISSUE INTERACTION}

\section{Properties of light}

A beam of light is made up of countless trillions of photons, and each photon is a weightless packet of pure energy. Light can be reflected off tissue, particularly the stratum corneum, transmitted into tissue to reach targets in various layers, scattered forwards, laterally and backwards by the many optically different components which make up skin, and ultimately absorbed in the target tissue. All of these occur together to some degree when a beam of light energy strikes skin, but the most important is absorption because the first law of photobiology is very clear: if light is not absorbed in a target, then there will be no reaction. Absorption comprises an energy exchange from the photon to the absorbing element or target, often referred to as the chromophore.

\section{Photothermal reactions in tissue}

The rate of the energy exchange depends on the incident power of the beam: the higher the power, the greater will the energy exchange be, resulting eventually in the production of a light-heat energy transfer: the photothermal effect. Lasers have an intrinsically high photon intensity compared with light-emitting diodes (LEDs), for example, so laser energy became a natural photosurgical resource. Fig. 1 illustrates schematically what happens when a beam of laser energy with high incident power strikes tissue and is absorbed.
Irreversible damage

(photothermal)

Reversible changes (photothermal)

Cellular activation (athermal, atraumatic)
Fig. 1. Schematic showing simultaneous range of photothermal reactions and tissue temperature ranges associated with a carbon dioxide $\left(\mathrm{CO}_{2}\right)$ laser imact, from photodestructive to photobioactivative. 
A range of photothermally-mediated reactions occurs depending on the temperature created in the target, from ablation/vaporization and carbonization, through coagulation to protein denaturation, Because not all of the incident photon energy is taken up with the ablation process, especially with the longer pulse widths, gradually decreasing photon intensities penetrating into the tissue result in lower temperatures and the zones of different photoeffects. However, at the periphery of the photodestructive effects there is a very interesting zone of little or no temperature rise at all, associated with an athermal effect and without any damage. It is a scientific rule that the energy from the photons must go somewhere, and it goes directly into the energy pool of the target cells thereby raising their energy levels athermally and atraumatically thus resulting in photobiomodulation of the cells' activities. ${ }^{9,10}$ This potentially therapeutic reaction occurs simultaneously with the photodestruction occurring at higher temperatures. The early adopters of the surgical $\mathrm{CO}_{2}$ all reported lower postoperative pain and inflammation with $\mathrm{CO}_{2}$ procedures than the same procedure carried out with the conventional scalpel, and this simultaneous phototherapy was why this phenomenon occurred. ${ }^{11}$

\section{The importance of wavelength}

It can be argued that the most important parameter for any light-based medical system is not the power of the beam, but its wavelength, which is a direct component of the particle/waveform duality of photons, Wavelength determines above anything else what the target of the treatment beam is, the chromophore, and also controls the intrinsic absorption of the beam into living tissue, more so than does the beam power. ${ }^{12}$ Fig. 2 illustrates the absorption spectra from the visible waveband at $400 \mathrm{~nm}$ to 700 $\mathrm{nm}$, to mid-infrared (IR) out to $11.2 \mu \mathrm{m}$ for the biologic chromophores of melanin, hemoglobin, and tissue water with a selection of laser wavelengths, with a photospectrogram of the penetration of 'white light' lequalized over $400 \mathrm{~nm}-1,100 \mathrm{~nm}$ ) through the author's hand. Each of these components of the figure helps to explain the other. Shorter wavelengths of visible light are heavily absorbed in melanin in the epidermis and blood in the dermis, hence the high optical density (OD) value of around 8 on the photospectrogram for blue light to yellow/orange, and poor penetration. Red light at $633 \mathrm{~nm}$, only $43 \mathrm{~nm}$ longer than $590 \mathrm{~nm}$ yellow, is less absorbed in melanin and considerably less absorbed in oxyhemoglobin, so penetration is several order of magnitude higher than the $590 \mathrm{~nm}$ yellow wavelength illustrated in the photospectrogram, bearing in mind that OD units have a logarithmic value. $830 \mathrm{~nm}$ is at the bottom of the water absorption curve, and is less absorbed in both blood and melanin so shows the optimum penetration. After 1,000 nm, absorption in tissue water starts to play an important component in determining decreasing penetration, and becomes a major chromophore around the 2,000 nm mark. Very high absorption in tissue water is seen at the $\mathrm{CO}_{2}$ laser wavelength of $10,600 \mathrm{~nm}$, and is one order of magnitude higher at 2,940 $\mathrm{nm}$, the wavelength of the Er:YAG laser. This predetermined the role of these lasers in ablative laser resurfacing, either full face or fractional. The lower absorption by tissue water of the $\mathrm{CO}_{2}$ wavelength allowed this laser to become the "light scalpel" with secondary coagulation of tissues and small blood vessels giving good hemostasis, compared with the Er:YAG which gives much more controlled ablation, but with much less coagulation and hence less hemostasis. Wavelength is therefore a very powerful determinant of the absorption component of the basic light/tissue reaction of light-based medical systems, ${ }^{13}$ determining not only the target chromophore

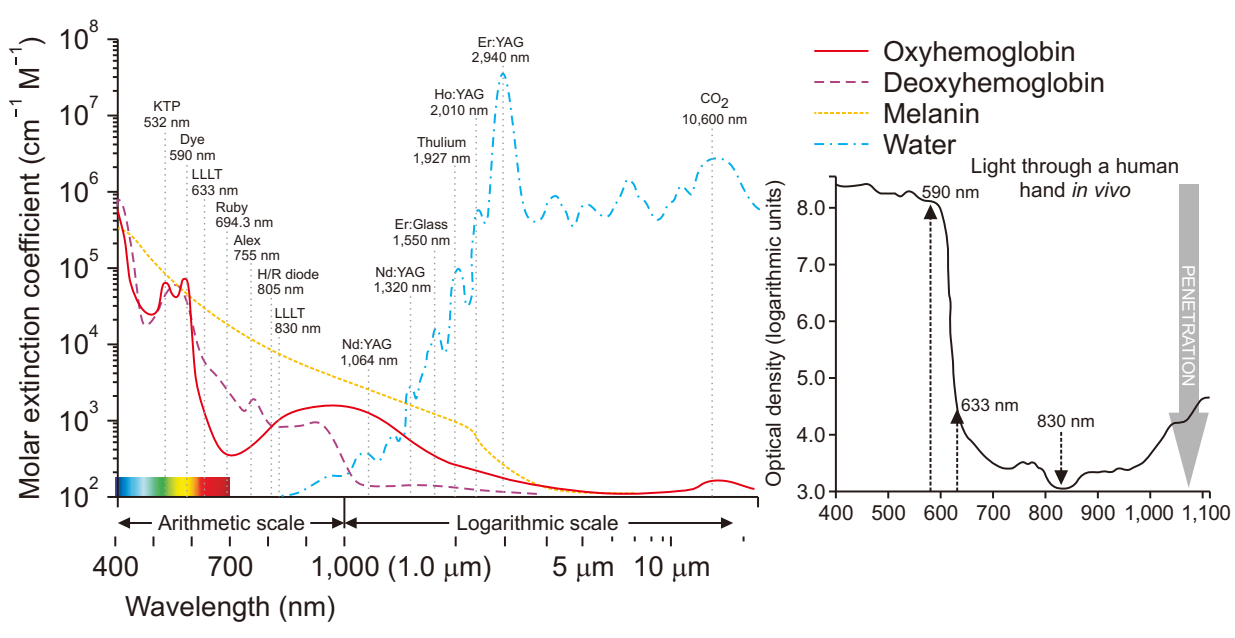

Fig. 2. (Main picture): Spectral responses of the hemoglobins, melanin and water with a selection of lasers, indicating where they intersect the biological chromophore curves. (Inset picture): Computergenerated photospectrogram of white light through a human hand in vivo. 
for any laser system but also how deep that laser can intrinsically penetrate into tissue.

\section{TREATMENT PARAMETERS: FIXED AND ADJUSTABLE}

The wavelength of a light-based source is usually fixed, so it is an inherent parameter: however, a very few systems offer selectable wavelengths from the one medium, such as 1,064 nm for the Q-switched Nd:YAG with $532 \mathrm{~nm}$ from frequency-doubling the beam plus a visible yellow and red wavelength with the addition of dye handpieces pumped by the $532 \mathrm{~nm}$ beam. However, these are the exceptions. The beam mode is also usually fixed as pulsed or continuous wave (CW). The parameters that are nearly always adjustable include the output power incident at the tissue measured in W, the spot size of the beam at the tissue or the treated area measured in centimeters $(\mathrm{cm})$, and the pulse width or exposure time measured in seconds (s). Table 2 lists the major units and prefixes seen in the laser and light-based literature. The pulse width of a light-based device should strictly speaking only apply to true pulsed lasers, i.e., where the width of the pulse is 1 ms or less and is often a fixed parameter, although some nanosecond/picosecond lasers offer selectable pulse widths. It is also important when thinking about lighttissue interaction to consider longer 'pulses' of light as delivered from switched or gated CW systems and from the newer long-pulsed dye lasers, and long-pulsed ruby, $\mathrm{Nd}: Y A G$ and alexandrite systems.

\section{Power, power density (irradiance) and energy density (fluence)}

Two of the three main adjustable parameters of any light based system are the output power and the spot size. From these can be derived the power density (PD) or irradiance. When considering any laser or non-laser system, the PD is the most important parameter of all, apart perhaps from wavelength as discussed above. ${ }^{12}$
Power density: the medicine: The PD of a laser is calculated by dividing the output power at the tissue in $\mathrm{W}$ by the irradiated area in $\mathrm{cm}^{2}$ (square centimeters). The irradiated area is derived by multiplying 2 contiguous sides of a rectangular beam expressed in $\mathrm{cm}$, or by using the formula $\pi \cdot r^{2}$ in a circular spot size where $\pi$ is the constant pi, 3.142, and $r$ is the radius (not the diameter) of the spot expressed in $\mathrm{cm}$. The PD is given in watts per square centimeter $\left(\mathrm{W} / \mathrm{cm}^{2}\right)$ with the appropriate prefix where required (see Table 2). By altering only the spot size of a laser through moving its beam into and out of focus, a huge range of PDs can be achieved which are orders of magnitude different, If a laser has a range of output power of from $1 \mathrm{~W}$ to $10 \mathrm{~W}$, that only allows one order of magnitude between the minimum and maximum power. However if that same laser at $2 \mathrm{~W}$ is focused to a spot size $100 \mu \mathrm{m}$ in diameter, the power density is actually $25,000 \mathrm{~W} / \mathrm{cm}^{2}$. That is enough to incise or excise tissue. The handpiece is moved away from the tissue to give a $200 \mu \mathrm{m}$ spot, but the power density drops to $6,250 \mathrm{~W} / \mathrm{cm}^{2}$, not one-half, but one quarter, and the beam can now be used for gentle ablation of tissue. If the spot size is further increased to 1.00 $\mathrm{mm}$, ten times the original, the PD drops dramatically to $250 \mathrm{~W} / \mathrm{cm}^{2}$, one-hundredth the original and a range of 2 orders of magnitude at which tissue can be coagulated. When defocused by a factor of 100 to $1.0 \mathrm{~cm}$, the PD is 2.5 $\mathrm{W} / \mathrm{cm}^{2}$ at which tissue will not be heated up very much, if at all ... in other words an athermal and atraumatic reaction associated with therapeutic rather than surgical effects. These data are illustrated in Fig. 3, and suggest that with the same $2 \mathrm{~W}$ beam, given that the wavelength is appropriate for the correct laser/tissue reaction (such as at $10,600 \mathrm{~nm}$ with the $\mathrm{CO}_{2}$ laser), it is possible to excise or incise tissue, then to draw back the handpiece to vaporize or debulk the target. If a small blood vessel starts to bleed, drawing back the handpiece further allows hemostasis through coagulation, and finally the wound healing of the target area can be accelerated with athermal low level laser therapy (LLLT). This is why the PD is a critical

Table 2. Common units used to describe laser parameters

\begin{tabular}{|c|c|c|c|c|c|c|c|c|}
\hline \multicolumn{3}{|c|}{ Power in watts (W) } & \multicolumn{3}{|c|}{ Size in centimeters $(\mathrm{cm})$} & \multicolumn{3}{|c|}{ Pulse/exposure in seconds } \\
\hline Terawatt & TW & $10^{12}$ & Centimeter & $\mathrm{cm}$ & $10^{-1}$ & Second & $s$ & \\
\hline Gigawatt & GW & $10^{9}$ & Millimeter & $\mathrm{mm}$ & $10^{-3}$ & Millisecond & $\mathrm{ms}$ & $10^{-3}$ \\
\hline Megawatt & MW & $10^{6}$ & Micrometer & $\mu \mathrm{m}$ & $10^{-6}$ & Microsecond & $\mu \mathrm{s}$ & $10^{-6}$ \\
\hline Kilowatt & KW & $10^{3}$ & Nanometer & $\mathrm{nm}$ & $10^{-9}$ & Nanosecond & ns & $10^{-9}$ \\
\hline Watt & W & & & & & Picosecond & ps & $10^{-12}$ \\
\hline Milliwatt & $\mathrm{mW}$ & $10^{-3}$ & & & & Femtosecond & fs & $10^{-15}$ \\
\hline Microwatt & $\mu \mathrm{W}$ & $10^{-6}$ & & & & & & \\
\hline
\end{tabular}




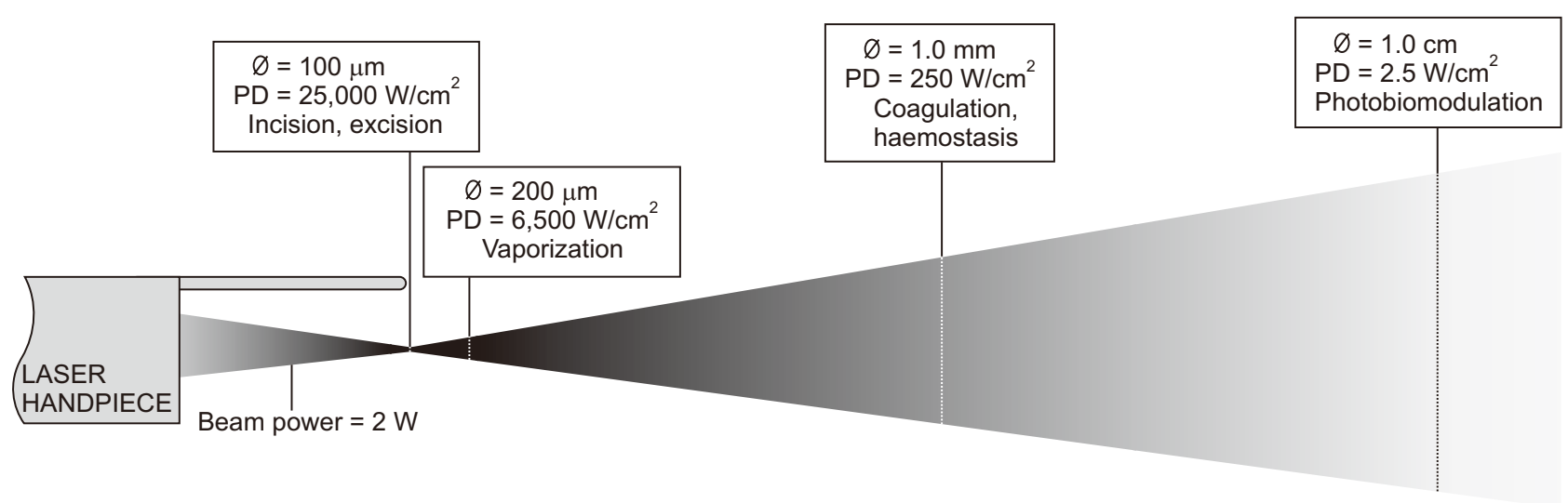

Fig. 3. The importance and versatility of power density (or irradiance) demonstrated for a $2 \mathrm{~W}$ CW laser: Simply moving into or out of the focal point enables an entire range of photoreactions.

Table 3. This illustrates a variety of bioeffects $(\Delta \alpha)$ achieved with the same approximate energy density, or dose, of $25 \mathrm{~J} / \mathrm{cm}^{2}$

\begin{tabular}{cccccccc}
\hline $\mathrm{P}$ & $\mathrm{S} \varnothing$ & {$[\mathrm{a}]\left(\mathrm{cm}^{2}\right)$} & $\mathrm{PD}\left(\mathrm{W} / \mathrm{cm}^{2}\right)$ & $\mathrm{t}$ & $\mathrm{e}$ & $\mathrm{ED}\left(\mathrm{J} / \mathrm{cm}^{2}\right)$ & $\Delta \alpha$ \\
\hline $100 \mathrm{~W}$ & $10.0 \mathrm{~cm}$ & 78.6 & 1.3 & $20 \mathrm{sec}$ & $2,000 \mathrm{~J}$ & 25 & - \\
$50 \mathrm{~W}$ & $3.5 \mathrm{~mm}$ & 0.1 & 500 & $100 \mathrm{msec}$ & $5 \mathrm{~J}$ & 25 & + \\
$10 \mathrm{~W}$ & $1.0 \mathrm{~mm}$ & 0.0008 & 1,250 & $20 \mathrm{msec}$ & $0.2 \mathrm{~J}$ & 25 & ++ \\
$1 \mathrm{~W}$ & $200 \mu \mathrm{m}$ & 0.0003 & 3,180 & $8 \mathrm{msec}$ & $8 \mathrm{~mJ}$ & 25 & +++ \\
$75 \mathrm{~mW}$ & $3.0 \mathrm{~mm}$ & 0.07 & 1.1 & $23 \mathrm{sec}$ & $1.725 \mathrm{~J}$ & 25 & - \\
\hline
\end{tabular}

As can be seen from the table, the power density (PD) is the most important determinant of the bioeffect from frank surgery to photobiomodulation, and the energy density alone is therefore not a real determinant of the ultimate surgical or therapeutic effect. Note also the uselessness of the energy in $\mathrm{J}$ in predicting the bioeffect: $8 \mathrm{~mJ}$ of energy at $1 \mathrm{~W}$ could create strong photosurgical damage, but $2,000 \mathrm{~J}$ at $100 \mathrm{~W}$ would not raise the target temperature at all, depending always on the PD.

Key to table: P: Incident power (units as shown). SØ: spot size diameter (units as shown). [a]: irradiated area (in $\mathrm{cm}^{2}$ ). PD: power density in W/ $\mathrm{cm}^{2}$. t: exposure time (units as shown). E: energy (units as shown). ED: energy density in $\mathrm{J} / \mathrm{cm}^{2} . \Delta \alpha$ : graded bioeffects ( +++ , severe photodestruction; ++, medium photodestruction; +, mild and/or reversible photodestruction; -, photobiomodulation (LLLT).

element when deciding the laser-tissue interaction.

Energy density: the dose: Adding the irradiation time or exposure time to the PD will allow the energy density (ED) of the beam to be calculated, also known as the fluence or the dose, expressed in joules per square centimeter $\left(\mathrm{J} / \mathrm{cm}^{2}\right)$. This gives us the energy delivered to the tissue quantified by the unit area irradiated. The ED is not to be confused with the energy alone in J, however, which is simply power over time and not really a useful treatment parameter. Having selected the power density to give the surgical effect desired, by then adding the irradiation time the clinician can control the degree or volume of that effect, to achieve a greater or a smaller reaction. However, the energy density is not the determinant of the basic surgical or therapeutic response: that is the role of the PD. This is illustrated in Table 3 where the same dose of $25 \mathrm{~J} /$ $\mathrm{cm}^{2}$ is associated with a variety of bioeffects from photodestruction to photobiomodulation. If the ED is the dose, then the PD can be likened to the medicine. As any clini- cian or pharmacist knows, if the medicine is incorrect, playing around with the dose will not achieve the desired result.

\section{Use the parameters to make science work for you}

An understanding of the photobiological relationship between the parameters and laser-tissue interaction is essential, and will make a good laser clinician into an excellent one. The $\mathrm{CO}_{2}$ laser is recognized as a surgical laser, and often classed as a 'high power' laser. A $50 \mathrm{~W}$ $\mathrm{CO}_{2}$ laser defocused to a $10 \mathrm{~cm}$ spot size, the area of a large crural ulcer, for example, gives a power density of just over $630 \mathrm{~mW} / \mathrm{cm}^{2}$. That is a phototherapeutic power density from a photosurgical laser, with no heating and no damage, and will help to heal the ulcer. The Er:YAG laser is associated with excellent but shallow ablation, very little secondary coagulation (residual thermal damage) of a few micrometers in depth, and poor hemostasis: this is why the $\mathrm{CO}_{2}$ laser is preferred for both full-face and frac- 
tional ablative laser resurfacing. However, if a single pass is made with the ablative mode of the Er:YAG, the epidermis will be cleanly ablated off the dermis, with very minor pinpoint bleeding as a good indicator that the dermis has been reached. The parameters are then altered to give a subablative effect with a larger spot size and a lower pulse energy, and several passes with overlapping are made through the epidermal window onto the exposed dermis: a coagulative effect will be achieved, similar to the $\mathrm{CO}_{2}$. Thus the benefit of the Er:YAG is maximized Iclean ablation of the epidermis) with the benefit of the residual thermal damage left after $\mathrm{CO}_{2}$ laser resurfacing managed by knowing and using parameter-mediated photobiology.

\section{Beam pulsewidth: from $\mathrm{CW}$ to picosecond domain}

Fig. 4 illustrates the waveform of different pulsewidths, although strictly speaking gating or chopping a CW beam does not produce true pulses. In a CW system (Fig. 4A), when the footswitch is depressed, the laser is fired and remains firing at the set output power until the footswitch is released. CW systems can be set to produce a chopped beam with exposure intervals separated by interexposure intervals where the laser beam is off. CW emission is measured in seconds, with the shortest exposure limited to around $100 \mathrm{~ms}$, or one-tenth of a second, with maximum power usually in tens of $\mathrm{W}$.

The original ruby laser produced a true pulse of $1 \mathrm{~ms}$ or less, but it was a ragged-looking pulse owing to impurities in the ruby crystal with its peak power at the beginning of the pulse, decaying with time (Fig. 4B). However, this peak power was much higher than in the case of the CW systems, measured in MW.

A way was found to 'tidy up' the quality of the cavity (denoted by Q) through a method called Q-switching, whereby the optical quality of the cavity is rapidly turned off by denying access of the energy in the cavity to one of the mirrors (which are essential for amplification by stimulated emission), and then restored. ${ }^{14}$ While the quality of the

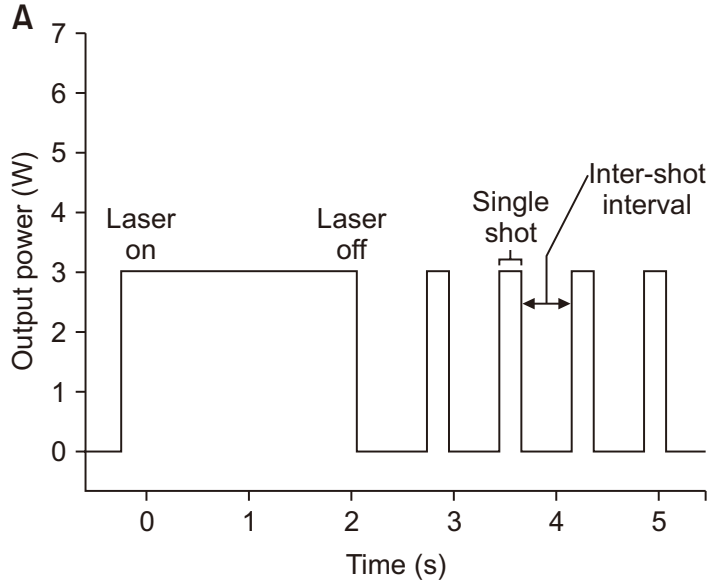

D

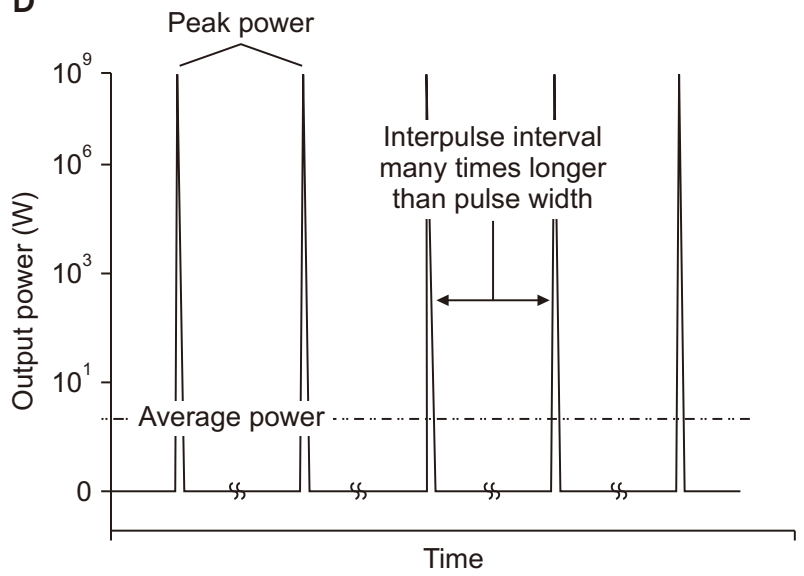

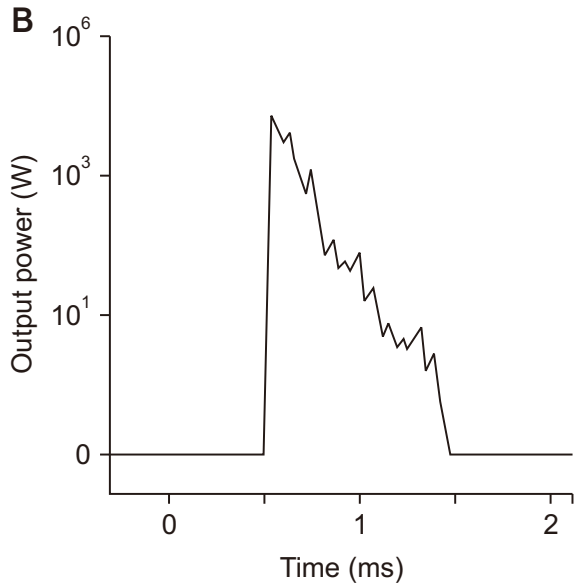

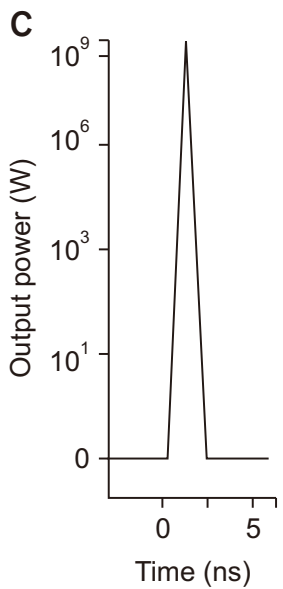

E

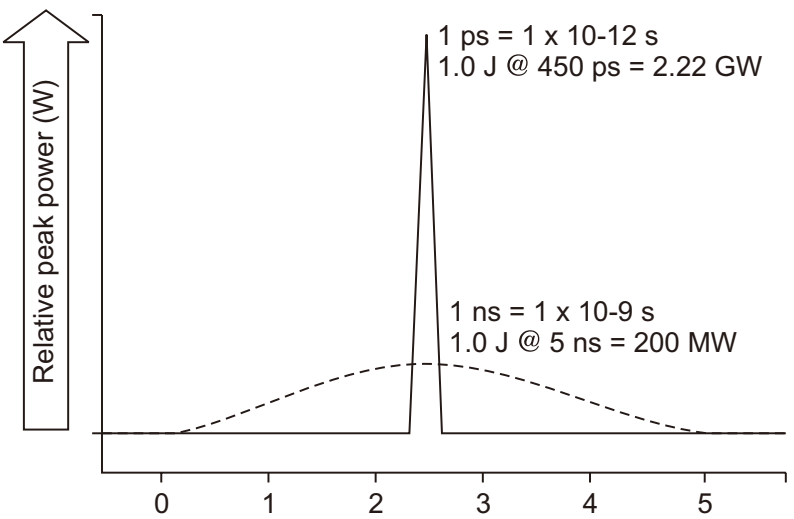

Fig. 4. Continuous wave and pulsed laser waveform illustrated. (A) CW and chopped CW beam. (B) 1 ms pulsed laser. (C) Single shot Q-switched laser beam. (D) Train of high-frequency Q-switched beams separated by a very long interpulse interval to give a beam 'seen' by the tissue as CW, hence 'quasi-CW'. (E) Not euro. $€$ : The significant difference in peak power between a $5 \mathrm{~ns}$ Q-switched beam and a $450 \mathrm{ps}$ beam. 
cavity is spoiled the pumping energy continues to build up the spontaneous emission within in the cavity, but it has nowhere to go as lasing cannot occur so the build-up of power in the cavity is immense. When the optical quality is suddenly restored to the cavity, the built-up power is released in a single spike of very high peak power, with the pulse width in the nanosecond domain (Fig. 4C). The original Q-switching was achieved mechanically with a rotating prism which alternately removed and restored the quality of the cavity, switching it on and off and hence called Q-switching. Modern Q-switching is achieved without any mechanical means giving shorter pulses in the single unit nanosecond domain. Not only did Q-switching tidy up the pulse characteristics, it also allowed significantly higher peak powers per pulse in the GW range. As a subset of the $Q$-switched laser, by creating a train of $Q$ switched pulses at an extremely high frequency separated by an interpulse interval orders of magnitude greater than the pulse width, each individual pulse is shorter than the thermal relaxation time of the target tissue, so instead of 'seeing' the train of $Q$-switched peaks, the tissue 'sees' the average power of the beam so the end result is delivery of power seen by the tissue in a range of watts similar to a CW output (Fig. 4D). This is termed quasi-CW, and was used to provide the superpulsed and ultrapulsed beams for $\mathrm{CO}_{2}$ lasers with extremely precise ablation and minimal charring. The next step was to design the cavity to deliver ultrashort pulses in the picosecond domain, and the picosecond laser (ps-laser) became commercially available. The effect of shortening the pulse width to the ps-domain allows the delivery of peak powers in the terawatt (TW) range (Fig. 4E). Table 4 shows the dramatic effect on the peak power of shortening the pulse width in a beam with the same energy density of $1 \mathrm{~J} / \mathrm{cm}^{2} \mathrm{com}$ pared for exposures in the $\mathrm{CW}$ range all the way to the psdomain. The benefits of the ultrashort high peak power beam are better target selectivity with less dependence on pigment selectivity, better target destruction with absolutely minimal damage to surrounding normal tissue.

\section{Selective photothermolysis vs super-selective mechanolysis}

The early days of laser photocoagulation depended on the creation of heat in the target tissue via absorption of the energy in the target chromophore. In the case of the visible light lasers, the target was blood, melanin, or some other abnormal pigment in the skin. In the case of the $\mathrm{CO}_{2}$ laser, it was tissue water. With the comparatively long exposure times available with the CW lasers (usually the minimum was $100 \mathrm{~ms}, 0.1 \mathrm{~s}$, per shot), as the target slowly heated up it lost heat to the surrounding normal tissue thus creating a wave of secondary conducted heat. In the case of the visible light lasers, the effect was at least semi-selective with destruction of the target chromophore but secondary coagulative damage to the surrounding normal tissue. In the case of the $\mathrm{CO}_{2}$ laser, all of the target tissue was affected because tissue water was the chromophore.

In 1983, Anderson and Parrish published the theory of selective photothermolysis, ${ }^{15}$ whereby using a laser with a wavelength matched to a pigmented target's action spectrum, and at a pulsewidth shorter than the thermal relaxation time (TRT) of the target, such as the pulse widths in the ns domain, gave an almost non-linear photothermal reaction with some photomechanical component which was confined to the target itself without spreading to the surrounding normal tissue. The target is destroyed, shattered into smaller fragments, while the surrounding normal tissue is left unharmed. Another possible descriptive term for this is thermal lock-in.

The delivery of ultrashort pulses in the ps domain creates a different reaction which is based on the stress relaxation time of the target, and the shortening of the pulse width removes to some extent the pigment selectivity which was the hallmark of the selective photothermolysis approach. ${ }^{16}$ The energy absorbed by the target stresses the target in a more nonlinear fashion than the ns-domain laser so that the buildup of energy locked within the target in the ultrashort ns pulse disintegrates the target into finer particles than the ns-domain laser, with no damage to surrounding normal tissue. The other possible name

Table 4. Demonstration of the dramatic influence on power density compared by pulse width and the same energy density

\begin{tabular}{ccrr}
\hline Exposure time (units as shown) & Energy density $\left(\mathrm{J} / \mathrm{cm}^{2}\right)$ & Peak power density (units as shown) & Terminology \\
\hline $1.0 \mathrm{~s}$ & $1.0 \mathrm{~J} / \mathrm{cm}^{2}$ & $1 \mathrm{~W} / \mathrm{cm}^{2}$ & watt $-\mathrm{W}$ \\
$1.0 \mathrm{~ms}$ & $1.0 \mathrm{~J} / \mathrm{cm}^{2}$ & $1,000 \mathrm{~W} / \mathrm{cm}^{2}$ & kilowatt $-\mathrm{kw}$ \\
$1.0 \mathrm{ss}$ & $1.0 \mathrm{~J} / \mathrm{cm}^{2}$ & $1,000,000 \mathrm{~W} / \mathrm{cm}^{2}$ & megawatt $-\mathrm{MW}$ \\
$1.0 \mathrm{~ns}$ & $1.0 \mathrm{~J} / \mathrm{cm}^{2}$ & $1,000,000,000 \mathrm{~W} / \mathrm{cm}^{2}$ & gigawatt $-\mathrm{GW}$ \\
$1.0 \mathrm{ps}$ & $1.0 \mathrm{~J} / \mathrm{cm}^{2}$ & $1,000,000,000,000 \mathrm{~W} / \mathrm{cm}^{2}$ & terawatt - TW \\
\hline
\end{tabular}


for this approach is the stress lock-in reaction.

At present systems are commercially available delivering 450 ps or 350 ps. However, these are not what I would call 'true' ps-lasers, because compared to a 5 ns Q-switched laser, the current ps-lasers are delivering 0.45 or $0.35 \mathrm{~ns}$. It is correct to say that the majority of the reaction locked within the target is photomechanical but there is still a photothermal element, and at the current ps pulsewidths 'color-blindness' has not yet been completely achieved. Multiple wavelengths are still therefore required when treating multicolor tattoos. However, once systems are available with pulsewidths in the tens of ps or even in single unit ps, then the picosecond laser will become much more like the magic wand which at present they are not. The ps laser is definitely still a work of art in progress.

\section{NON-LASER SYSTEMS}

So far I have talked mostly about lasers, but some attention has to be paid to the non-laser light-based sources, namely intense pulse light systems, and low level light therapy with light-emitting diode (LED)-based systems.

\section{Intense pulsed light}

Intense pulsed light (IPL) systems are based around a gas-filled flashlamp mounted in the system handpiece, usually xenon gas, pumped with a charge of high voltage electrical energy stored in a capacitor. ${ }^{17}$ Fig. 5 illustrates the basic components of any IPL system. IPLs deliver a powerful pulse of polychromatic light, extending from the near-IR to the shorter blue visible wavelengths, as shown in Fig. 6. Any ultraviolet light is filtered out before the light energy is delivered to the target tissue. IPL systems are often confused with lasers, but they deliver noncoherent

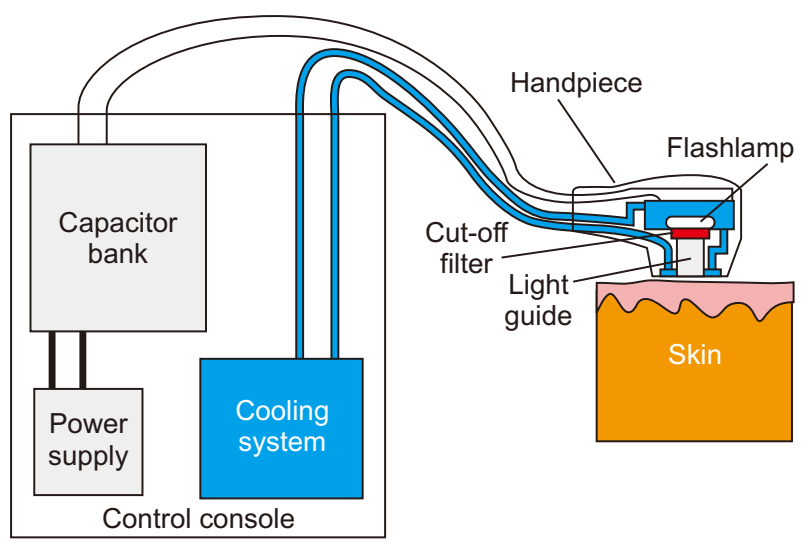

Fig. 5. The anatomy of a typical intense pulsed light (IPL) system. light. Some degree of target selectivity is obtained by using precise filters to 'cut off' the unwanted visible wavelengths. Typical filters might be as follows: blue at $420 \mathrm{~nm}$ (acne); green at $510 \mathrm{~nm}$ (pigmented dyschromia, vascular work); yellow at $590 \mathrm{~nm}$ (pigmented dyschromia, vascular lesions in darker skin types); red at $620 \mathrm{~nm}$ (fine vessels, hair removal in lighter skin); deep red at $670 \mathrm{~nm}$ (hair removal in darker skin types and skin rejuvenation); and near IR at $710 \mathrm{~nm}$ (skin rejuvenation).

What has to be remembered is that these are simply cut-off filters, and do not deliver monochromatic light: the $420 \mathrm{~nm}$ filter will cut off any wavelengths shorter than that, but the light energy still spans from the near-IR all the way to the $420 \mathrm{~nm}$ cut-off point, and every wavelength in between (Fig. 7). At the other IR end of the spectrum, most unfiltered IPL lamps produce some high peaks in the near-IR from the 1,400 nm point. These longer com-

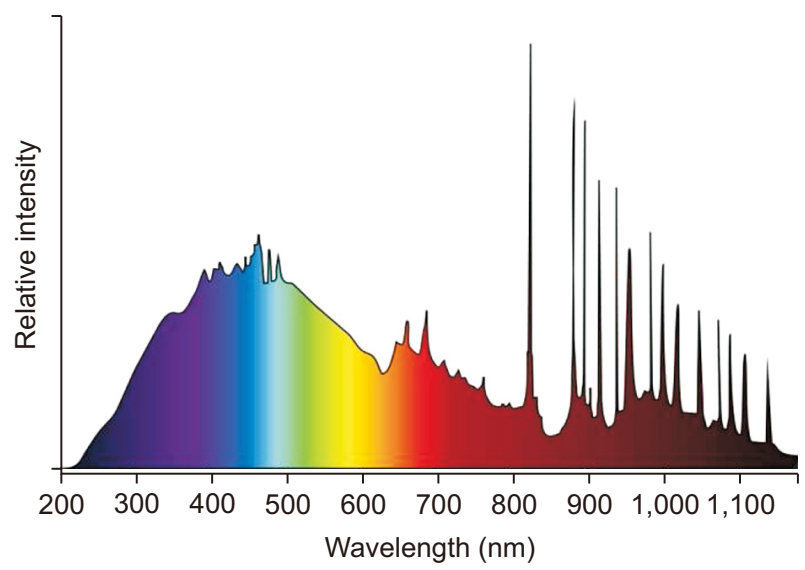

Fig. 6. Full spectrum of an IPL flashlamp discharge, from nearinfrared to ultraviolet.

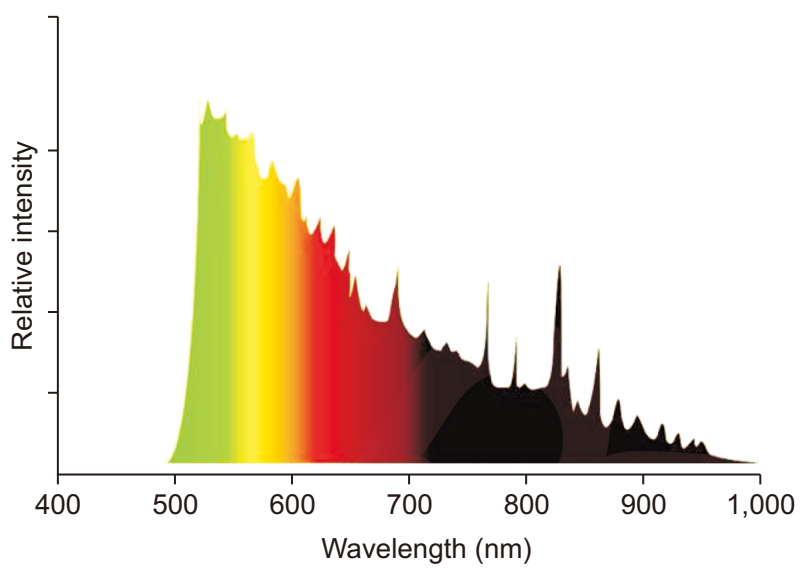

Fig. 7. Typical final polychromatic but semiselective beam from an IPL with a cut-off filter at $510 \mathrm{~nm}$ and a cut-on filter at $950 \mathrm{~nm}$. This could be used to treat vascular and pigmented lesions. 


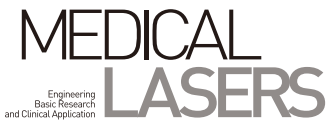

ponents of the near-IR waveband do nothing in tissue other than produce heat, so many systems incorporate a cut-on filter to limit the near-IR from $950 \mathrm{~nm}$ or so, where there is still some pigment selectivity with minimal heating.

IPL systems were originally very basic, but have benefitted from technological advances which have improved the characteristics of the beam produced. The features of the total discharge type of flashlamp, whereby all of the electrical energy stored in the device capacitor is released at once, impose a wavelength shift on the spectral output of the light produced by the lamp which moves over the period of the pulse from IR to blue and back to IR, known as spectral jitter. When a single pulse of light is actually composed of a train of from 2 to 5 pulses separated by an interpulse interval, this problem becomes worse. This is not the optimum distribution of energy delivered to the skin and can lead to unexpected results owing to the change in the spectral pattern of the light reaching the skin. Other systems use a different technology whereby the flashlamp is continuously kept in a simmer mode. When the electrical charge is generated by the system to fire the flashlamp it produces time resolved spectral distribution over the duration of the pulse which eliminates the problem caused by spectral jitter. ${ }^{18}$ Older systems, and some newer ones with older technology, produce a more Gaussian-looking beam with hotspots. This will obviously create an uneven tissue response with the development of possible hotspots and tissue damage. This problem is reolved with square bean technology which delivers the light energy homogeneously over the treated area under the light guide. ${ }^{19}$ Most commercially available IPL systems benefit from one or the other of thse technologies, but only a very few offer both.

IPL systems in the hands of trained experts can produce excellent and safe results, and because of the wide range of indications, IPL systems are favoured by smaller clinics who perhaps do not have the budget to buy a number of laser systems with appropriate wavelengths. On the other hand, IPL systems are often found in the beauty salon market, being operated by therapists with insufficient training and therefore pose a potentially serious safety problem.

\section{Light-emitting diode-low level light therapy}

Referring back to Fig. 1, the outermost zone in that figure depicts an area of athermal and atraumatic lighttissue reactions which occur simultaneously with the photosurgical damage zones, and which serve to photoactivate the cells in that tissue to help with wound healing and repair. When it was discovered that it was the 'L' (light) of laser energy that was allowing early adopters of the $\mathrm{CO}_{2}$ surgical laser to experience less inflammation and oedema compared with the same procedure performed by the conventional scalpel, clinicians' and researchers' thoughts turned towards developing systems which could achieve only that photobiomodulatory activity without any heat or damage. Reports appeared as early as the late 1960s and early 1070s about the successful treatment of nonhealing crural ulcers with a very low output 10 mW HeNe laser from the late Professor Endré Mester working in Semmelweis University, Budapest. ${ }^{20,21}$ Mester called this effect 'laser biostimulation', and it heralded the beginning of dedicated laser systems being used for wound healing and pain attenuation throughout the 1970s into the 1980s. However, the science behind the practice was lacking so credibility was very low.

The HeNe lasers were very low-powered, so that treatment times to deliver useful doses were very long. To obtain more efficient treatment with higher power densities, defocused surgical lasers were often used for laser therapy. A 3 W CW Nd:YAG at 1,064 nm proved very effective in pain attenuation used with spot sizes of $2 \mathrm{~cm}$ in diameter or larger. At $2 \mathrm{~cm}$ in diameter, the power density of the laser enters the $\mathrm{mW} / \mathrm{cm}^{2}$ level $\left(955 \mathrm{~mW} / \mathrm{cm}^{2}\right)$. The literature suggests that PDs can go as high as around $5 \mathrm{~W} / \mathrm{cm}^{2}$ without inducing any temperature rise over a prolonged period of exposure. ${ }^{22}$ PDs over that level over longer exposures will induce some form of photothermal reaction, which does not fulfill the criteria of laser therapy as an athermal and atraumatic reaction. PDs of under $5 \mathrm{~W} / \mathrm{cm}^{2}$ can therefore be regarded as purely phototherapeutic.

Low level laser therapy: Eventually in the late 1970s and early 1980s, diode laser-based systems were also used, with $830 \mathrm{~nm}$ becoming a popular wavelength, and supplanting the stalwart $632.8 \mathrm{~nm}$ of the HeNe laser. ${ }^{23}$ Finally in 1988 the term 'low level laser therapy' (LLLT) appeared in the literature, coined by myself. ${ }^{24}$ LLLT is defined as a direct energy exchange between photons absorbed by the target cell which boost the cell energy pool, photoactivating the cell.

The basic process of photoactivation as defined by the eminent photobiologist Tiina Karu of the Moscow Academy of Sciences involves absorption of photon energy by a cell followed by signal transduction and amplification within the cell, resulting in the photoresponse. ${ }^{14}$ Visible light elicits a photochemical response through activation of the mitochondria within the cell, specifically though cytochrome c oxidase ( $\mathrm{CcO}$ ) at the end of the mitochondrial respiratory chain. This induces production of adenosine 
Photoreception $\longrightarrow$ Photoresponse

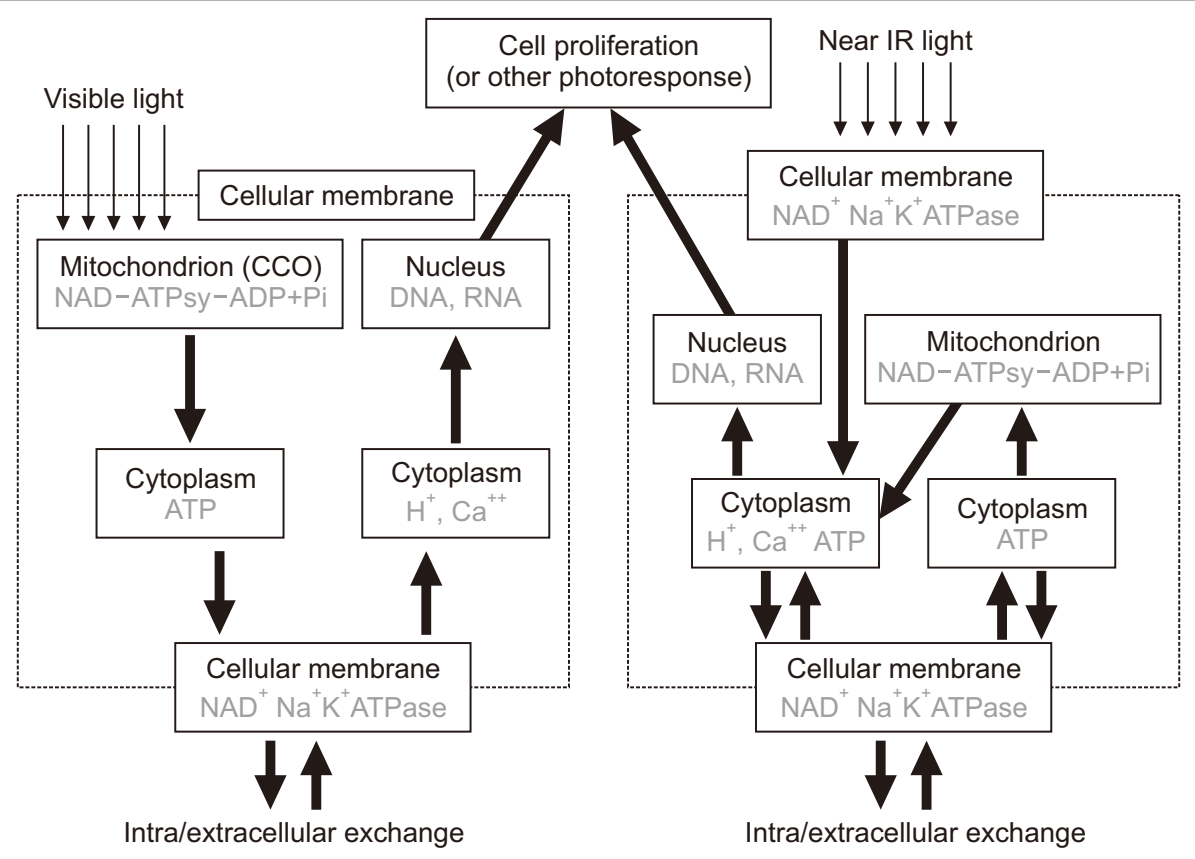

Fig. 8. The photochemical cellular response to a beam of visible light at low level light therapy levels (left panel) compared with the photophysical response of near infrared light (right panel). Despite the different primary response, the end result is the same: a photoactivated cell. triphosphate (ATP), fuel for the cell and for the organism. Near-IR light on the other hand induces a photophysical response in the cell membrane, finally resulting in ATP production from the mitochondria. The end result of both visible and near-IR light is therefore the same: a photoactivated cell. ${ }^{25}$ These concepts are illustrated in Fig. 8. If the photoactivated cell is damaged or compromised, it will be repaired. If it has a job to do, it will do it better, and if there are not enough of a particular cell type, others will be recruited in or proliferation will occur, One, two or all three things can happen in an LLLT-treated area.

Enter the light-emitting diode: Diode laser LLLT was extremely popular in the 1990s and proved effective particularly for wound healing and pain attenuation, particularly in Russia, Japan and Korea, and to a lesser extent in Australia and the UK, however application was almost always manually with a hand-held probe of some kind, point by point, and could be very clinician-intensive. Lightemitting diodes (LEDs) were suggested as possibly being able to be mounted in probes or arrays, but LEDs at that time were certainly cheap and cheerful but had low and unstable output powers, were highly divergent and had a wide waveband often spanning tens of $\mathrm{nm}$. It was easy to find a red LED, but almost impossible to source a narrowband $633 \mathrm{~nm}$ LED. This changed in 1998, with the development in the US NASA Space Medicine Laboratory of a new generation of LEDs, nicknamed the 'NASA LED'. ${ }^{25}$

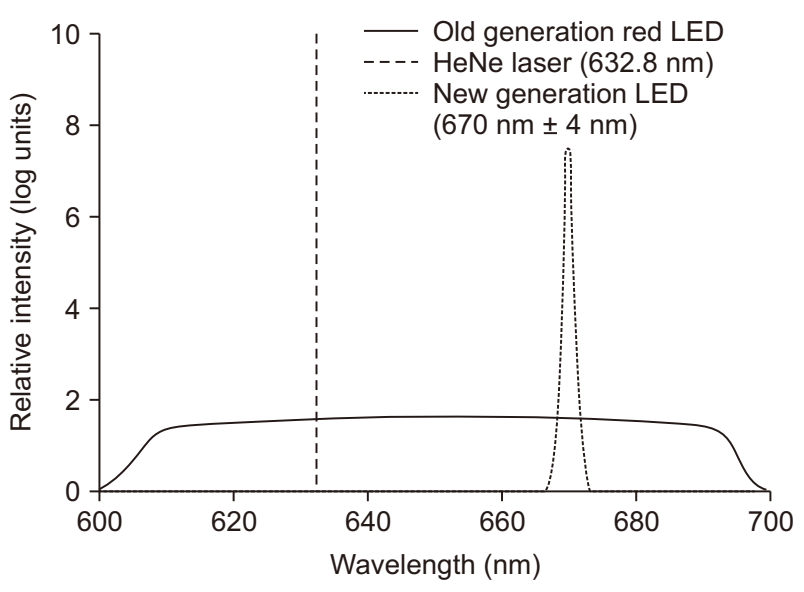

Fig. 9. The differences between the spectral output of an old generation red light emitting diode (LED), a HeNe laser and a new generation $670 \mathrm{~nm}$ visible red LED.

These had output powers 5 orders of magnitude better than existing LEDs, had a stable output, were much less divergent and, although not monochromatic like lasers, had good quasimonochromaticity enabling laser-like target selectivity (Fig. 9). A new and viable source had joined the phototherapy armamentarium. ${ }^{26}$

The new generation LEDs have strong inherent advantages over other light sources:

- They are very efficient, requiring a small DC input to 
generate a lot of light and therefore with low running costs.

- They can be mounted in large planar arrays, enabling irradiation of large areas of the body, like the face, in one session and in a hands-free manner. This makes them very therapist nonintensive.

- Because they are solid-state diodes they have no filaments nor need flashlamps. They therefore generate very little heat as a source of energy loss.

- They have powerful and stable output powers, capable of delivering strong photon intensity to the target tissue.

- They are quasimonochromatic, and although they are noncoherent, over $98 \%$ of the delivered photons are at the rated wavelength giving excellent and laser-like target selectivity.

- LED-LLLT is safe, effective easy to apply, pain- and side-effect free and is well tolerated by patients of all ages, from infants to centenarians.

In the past 15 years, more and more articles are appearing in the literature on the efficacy of LED-LLLT. If a PubMed search is run using LLLT as the keyword, over 4,000 hits will appear and a growing percentage of these are for LED-LLLT. Now the acronym is still LLLT, but it stands for 'low level LIGHT therapy', because LEDs have made such an impact in many medical specialities. ${ }^{27}$

One of the most popular indications has proved to be wound healing of all types of wounds, acute or chronic, ulcers, burns and surgical wounds. ${ }^{28}$ Both soft and hard tissues respond well with acceleration of the wound healing time. Inflammation is resolved quickly, and infection, both bacterial and viral, is controlled. ${ }^{29}$ Pain attenuation is a powerful indication with success reported in acute and chronic pain, arthroses, neuralgias, and herpes zoster. ${ }^{30}$ Photorejuvenation with LED-LLLT is proving very popular. ${ }^{31,32}$ Sports medicine is another fast-growing field, and one study reported that return to play was halved in injured athletes treated with $830 \mathrm{~nm}$ LED-LLLT compared with best standard care. ${ }^{33}$ The most popular wavelength for all indications appears to be $830 \mathrm{~nm}$ because of its deep penetration as seen in Fig. 2 above, and also because of the different cell types in the wound healing cycle which respond best to $830 \mathrm{~nm}$ compared with other wavelengths. ${ }^{34}$ Although very powerful on its own, LED-LLLT becomes even more interesting when it is used in combination with any other aesthetic or cosmetic approach. The synergy between the treatments ensures a better result faster, with satisfied patients. LED-LLLT is here to stay, and is constantly growing in indications together with a solid body of good scientific evidence.

\section{CONCLUSIONS}

Photosurgery and photomedicine have dramatically expanded in a pan-speciality way in the almost 6 decades since the first laser was successfully oscillated in 1960, and can be delivered with laser and non-laser devices. Medical lasers emit coherent, monochromatic light, and can precisely incise, excise, vaporize and coagulate tissue: however, they are also capable of producing therapeutic levels of incident light with correct parameters, i.e. athermal and atraumatic LLLT, the original low level laser therapy. Depending on a short or ultrashort pulse width and appropriate parameters they can satisfy the criteria of selective photothermolysis, or selective mechanolysis, and selectively remove pigmented targets. Intense pulsed light systems emit polychromatic and noncoherent light, which can be modulated with cut-off filters to give semiselectivity, and therefore do not totally fulfil the criteria of selective photothermolysis, but can be used for a range of indications including photorejuvenation, removal of dyschromia and small vascular lesions, and hair removal. LEDs emit quasimonochromatic but noncoherent light at very low incident power densities, and are therefore only capable of being used for LLLT, low level light therapy, for a vast range of wound healing and pain attenuation applications, as well as photorejuvenation. It is important to realize that all of these systems depend on the properties of light, and therefore in order to use them appropriately with safety and efficacy, the clinician must fully understand the complex photobiological principles governing light-tissue interaction. Based on this understanding, the expert clinician will be able to use these systems in a synergistic combination, because combination, together with the photobiological understanding, is the key to maximizing the enormous potential of photosurgery and photomedicine as a work of art, ars medicinae, still in progress.

\section{REFERENCES}

1. Fine S, Maiman TH, Klein E, Scott RE. Biological effects of high peak power radiation. Life Sci 1964;3:209-22.

2. Einstein A. Zur quantentheorie der strahlung. Physikalische Zeitschrift 1917;18:121-8.

3. Saleh BEA, Teich MC. Fundamentals of photonics. New York: John Wiley \& Sons; 1991.

4. Goldman L. Laser medicine in America: an overview. Lasers Surg Med 1981;1:285-8.

5. Townes $\mathrm{CH}$. Optical masers and their possible applications to biology. Biophys J 1962;2:325-9. 
6. Goldman L. Laser history and theory. In: Goldman L, editor. Biomedical aspects of the laser : the introduction of laser applications into biology and medicine. New York: Springer-Verlag; 1967. p.1-8.

7. Kaplan I. The CO2 surgical laser. Photomed Laser Surg 2010;28:847-8.

8. L'Esperance FA Jr. Historical aspects of ophthalmic lasers. Caduceus 1989;5:4-23.

9. Smith KC. The science of photobiology. New York: Plenum Press; 1977.

10. Karu T. Primary and secondary mechanisms of action of visible to near-IR radiation on cells. J Photochem Photobiol B 1999;49:1-17.

11. Ohshiro T. New classification for single-system light treatment. Laser Ther 2011;20:11-5.

12. Calderhead RG. Laser parameters. In: Calderhead RG, editor. Photobiological basics of photosurgery and phototherapy. Seoul: Hanmi Medical; 2011. p.57-61.

13. Karu TI, Kolyakov SF. Exact action spectra for cellular responses relevant to phototherapy. Photomed Laser Surg 2005;23:355-61.

14. Hardway GA. "Q" switching. Ann N Y Acad Sci 1965;122:608-13.

15. Anderson RR, Parrish JA. Selective photothermolysis: precise microsurgery by selective absorption of pulsed radiation. Science 1983;220:524-7.

16. Liu T, Wang J, Petrov GI, Yakovlev W, Zhang HF. Photoacoustic generation by multiple picosecond pulse excitation. Med Phys 2010;37:1518-21.

17. Raulin C, Goldman MP, Weiss MA, Weiss RA. Treatment of adult port-wine stains using intense pulsed light therapy (PhotoDerm VL): brief initial clinical report. Dermatol Surg 1997;23:594-7.

18. Ash C, Town G, Bjerring P. Relevance of the structure of timeresolved spectral output to light-tissue interaction using intense pulsed light (IPL). Lasers Surg Med 2008;40:83-92.

19. Mohanan S, Basheerahmed P, Priyavathani R, Nellainayagam G. New intense pulse light device with square pulse technology for hirsutism in Indian patients: a pilot study. J Cosmet Laser Ther 2012;14:14-7.

20. Mester E, Szende B, Spiry T, Scher A. Stimulation of wound healing by laser rays. Acta Chir Acad Sci Hung 1972;13:315-24.

21. Mester AF, Mester A. Wound healing. Laser Ther 1989;1:7-15.
22. Afrin N, Zhou JH, Zhang YW, Tzou DY, Chen JK. Numerical simulation of thermal damage to living biological tissues induced by laser irradiation based on a generalized dual phase lag model. Numer Heat Transf Appl 2012;61:483-501.

23. Calderhead RG, Ohshiro T. The Nd-YAG and GaAlAs lasers; a comparative analysis in pain therapy. In: Atsumi K, Nimsakul N, editors. Proceedings of laser Tokyo '81. Tokyo: Japan Medical Laser Society Society; 1982. Section IV, 1-4.

24. Ohshiro T, Calderhead RG. Low level laser therapy: a practical introduction. Chichester, UK: Wiley; 1988.

25. Smith KC. Laser (and LED) therapy is phototherapy. Photomed Laser Surg 2005;23:78-80.

26. Whelan HT, Houle JM, Whelan NT, Donohoe DL, Cwiklinski J, Schmidt MH, et al. The NASA light-emitting diode medical program-progress in space flight and terrestrial applications. Space Tech Appl Int Forum 2000;504:37-43.

27. Smith KC. Laser and LED photobiology. Laser Ther 2010;19:728.

28. Lee GY, Kim WS. The systemic effect of 830-nm LED phototherapy on the wound healing of burn injuries: a controlled study in mouse and rat models. J Cosmet Laser Ther 2012;14:107-10.

29. Min PK, Goo BL. 830 nm light-emitting diode low level light therapy (LED-LLLT) enhances wound healing: a preliminary study. Laser Ther 2013;22:43-9.

30. Park KY, Han TY, Kim IS, Yeo IK, Kim BJ, Kim MN. The effects of $830 \mathrm{~nm}$ light-emitting diode therapy on acute herpes zoster ophthalmicus: a pilot study. Ann Dermatol 2013;25:163-7.

31. Lee SY, Park KH, Choi JW, Kwon JK, Lee DR, Shin MS, et al. A prospective, randomized, placebo-controlled, double-blinded, and split-face clinical study on LED phototherapy for skin rejuvenation: clinical, profilometric, histologic, ultrastructural, and biochemical evaluations and comparison of three different treatment settings. J Photochem Photobiol 2007;88:51-67.

32. Calderhead RG, Vasily DB. Low level light therapy with lightemitting diodes for the aging face. Clin Plast Surg 2016:43:54150.

33. Foley J, Vasily DB, Bradle J, Rudio C, Calderhead RG. 830 nm light-emitting diode (led) phototherapy significantly reduced return-to-play in injured university athletes: a pilot study. Laser Ther 2016;25:35-42.

34. Kim WS, Calderhead RG. Is light-emitting diode phototherapy (LED-LLLT) really effective? Laser ther 2011;20:206-15. 